\title{
Factores de riesgo asociados con placenta retenida en ganado lechero en el norte de México
}

\section{Risk factors associated with retained placenta in dairy cattle in northern Mexico}

\author{
José María Carrera-Chávez ${ }^{1}$, Carla América Marmolejo-Aldana1 ${ }^{\circledR}$, Angélica María Escárcega-Ávila1@ Mateo

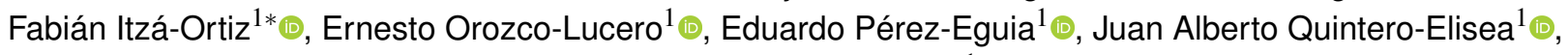 \\ Andrés Quezada-Casasola1@
}

\footnotetext{
${ }^{1}$ Departamento de Ciencias Veterinarias, Instituto de Ciencias Biomédicas, Universidad Autónoma de Ciudad Juárez. Anillo Envolvente y Estocolmo s/n. CP. 32310. Ciudad Juárez, Chihuahua, México.

*Autor de correspondencia: mateo.itza@uacj.mx
}

Nota científica recibida: 11 de diciembre de 2018 aceptada: 10 de junio de 2019

RESUMEN. El objetivo fue determinar la asociación entre diferentes factores de riesgo para retención placentaria (RP) en el norte de México. Se utilizaron registros de 2492 vacas Holstein de un establo comercial en Ciudad Juárez, Chihuahua, México. Las variables se analizaron mediante la prueba de ji-cuadrada $\left(\chi^{2}\right)$ y se calcularon las razones de momios (RM) para determinar la asociación de los factores de riesgo con RP. La incidencia total de RP fue del 10.15\%. Los factores de riesgo asociados con la RP fueron nacimiento gemelar y nacimiento prematuro, seguidos por mortinatos y distocia. La presencia de RP no aumentó el número de inseminaciones, pero disminuyó los días en leche en las vacas afectadas ( $p$ $<0.05$ ). En conclusión, los factores de riesgo de parto gemelar, parto prematuro, mortinatos, distocia y edad de la vaca afectan la incidencia de RP, mientras que temporada de parto no predispone a la presentación de esta alteración.

Palabras clave: Factores asociados, parámetros reproductivos, placenta retenida, trastornos reproductivos, vacas lecheras.

ABSTRACT. The objective was to determine the association between different risk factors for placental retention (PR) in northern Mexico. Records of 2492 Holstein cows from a commercial dairy herd located in Ciudad Juárez, Chihuahua, Mexico were used. Variables were analyzed by the chi-square test $\left(\chi^{2}\right)$ test and odds ratios $(\mathrm{OR})$ were calculated to determine the association of the risk factors with PR. The total incidence of PR was $10.15 \%$. The risk factors associated with RP were twin birth and premature birth, followed by stillbirths and dystocia. The presence of RP did not increase the number of inseminations, but decreased the days in milk in affected cows $(p<0.05)$. In conclusion, the risk factors twin birth, premature birth, stillbirth, dystocia and age of the cow affect the incidence of RP, while the delivery season does not predispose to the presentation of this alteration.

Key words: Associated factors, reproductive parameters, retained placenta, reproductive disorders, dairy cows.

\section{INTRODUCCIÓN}

La retención placentaria $(\mathrm{RP})$ es una falla de la expulsión de las membranas fetales. En vacas, se considera RP cuando las membranas fetales aún no han sido expulsadas entre 12 y 15 h post-parto (Sheetal et al. 2015, Sharma et al. 2017) y puede estar presente hasta por siete días post-parto o más si no se administra tratamiento (Moretti et al. 2015). Pero la etiología de este trastorno no está completamente entendida y la incidencia de RP en el ganado lechero va de 4 a 18\% (Hossain et al. 2015, Ku- mari et al. 2015). Los factores de riesgo establecidos para RP en regiones templadas incluyen, entre otros, el aborto, la muerte fetal, los partos gemelares, los partos prematuros, la distocia e inducción del parto (Hossain et al. 2015, Kumari et al. 2015). Otro factor que se ha asociado es el número de partos de las hembras, vacas de primera paridad son más propensas a presentar distocia, mientras que las vacas multíparas tienen más probabilidades de sufrir RP (Grohn y Rajala 2000). Aunque se ha sugerido que la ocurrencia de RP se puede disminuir mediante la manipulación de dichos factores de riesgo (Hossein- 
Zadeh y Ardalan 2011), la presentación de RP es multifactorial, ya que incluso en condiciones normales de gestación y parto, la tasa de RP es de aproximadamente $4.1 \%$ (LeBlanc 2008).

La RP es uno de los desórdenes reproductivos más comunes, ya que es una alteración que afecta a vacas reproductoras y repercute en la producción de leche de los bovinos, trayendo como consecuencia pérdidas económicas importantes (Dawod et al. 2015, Sheetal et al. 2015). El impacto de la RP varía desde nulo, hasta la disminución del comportamiento reproductivo. Esto se debe a que las vacas son más propensas a desarrollar metritis o endometritis (Barajas et al. 2018), tienen un intervalo más largo postparto hasta la primera ovulación y hay posibilidades que no haya gestación en sus próximos servicios (Hossain et al. 2015, Sharma et al. 2017), e incrementa el riesgo de pérdida fetal temprana (Mellado et al. 2016). Además, el tratamiento de alteraciones reproductivas como RP, metritis o endometritis implica la utilización de antibióticos, y en consecuencia, la leche de las vacas tratadas para consumo humano debe desecharse (Córdova-Izquierdo et al. 2017). Lo anterior indica la necesidad de identificar los factores que inciden en la presentación de esta alteración para controlar y evitar el problema (Hossein-Zadeh y Ardalan 2011). Además, se considera que mediante la aplicación de medidas que permitan el control de la incidencia de RP, otras alteraciones reproductivas pueden ser prevenidas (Melendez et al. 2006). Por lo anterior, el objetivo del presente trabajo fue determinar la relación que tienen diversos factores de riesgo con la incidencia de RP en un establo lechero del norte de México.

\section{MATERIALES Y MÉTODOS}

\section{Área de estudio}

El estudio se realizó en un establo lechero comercial de bovinos de raza Holstein ubicado en Ciudad Juárez, Chihuahua, México, localizado a los $31^{\circ} 35^{\prime} 54^{\prime \prime}$ LN y $106^{\circ} 19^{\prime} 27^{\prime \prime}$ LO. El clima de la zona es desértico, a lo largo del año hay ausencia de lluvias, siendo julio el mes más caluroso y enero el más frío. Los animales estuvieron en es- tabulación en corrales con cubículos de acceso libre, los cuales cuentan con sombra disponible para todos los animales, cada corral cuenta con un sistema de enfriamiento consistente en aspersores en el área de comederos y ventiladores con aspersión en el área de sombra.

La alimentación fue balanceada por el nutriólogo a cargo del establo en una ración mezclada de acuerdo con los requerimientos propios de la etapa fisiológica específica con base a las recomendaciones del National Research Council. Los primeros 21 días previos al parto la dieta de las vacas consistió en una mezcla de heno de avena, alfalfa, silo de sorgo, y pre-mezcla de grano de maíz molido con minerales y vitaminas. Las vacas se ordeñaron tres veces al día. El manejo reproductivo incluyó inseminación artificial, utilizando semen de empresas comerciales internacionales. Se detectó el estro dos veces al día y las vacas identificadas en estro se inseminaron de acuerdo con el esquema A.M./P.M. El diagnóstico de gestación se realizó por medio de ultrasonido con transductor rectal, modo $\mathrm{B}$, sonda lineal de $7.5 \mathrm{MHz}$ (Easi-Scan, BCF Technology) a los 35 días después de la inseminación y la gestación fue confirmada por palpación rectal 25 días después de realizar el ultrasonido. En caso de diagnosticar visualmente $R P$, las vacas se trataron con penicilina G procaína (200 $000 \mathrm{UI}$ ) y dihidroestreptomicina base (164 mg) a dosis de $20000 \mathrm{Ul} \mathrm{kg}^{-1}$ cada $48 \mathrm{~h}$ tres veces (Shotapen Classic, Virbac).

\section{Recolección de datos}

Se utilizaron registros de 2492 vacas de la raza Holstein con diferente número de lactancias; las vacas tenían entre tres y siete años de edad. Las fechas de parto ocurrieron del 1 de septiembre del 2015 al 30 de septiembre del 2016. Los datos fueron recolectados de la base de datos que se maneja en el establo (DairyQuest, Profitsource). La información correspondió a partos prematuros, partos gemelares, distocias, muertes peri-natales, retención placentaria, época del año, número de servicios por concepción, número de lactancias y días en leche (DEL). Las épocas climáticas de parto se clasificaron en otoño (octubre a noviembre), invierno (diciembre a marzo), 
primavera (abril a junio) y verano (julio a septiembre).

\section{Análisis estadístico}

Este estudio fue de tipo observacional analítico retrospectivo. Las variables cualitativas que se incluyeron para el análisis de asociación con la RP fueron: partos prematuros, partos gemelares, distocias, muertes peri-natales y época del año. Inicialmente se calculó el estadístico ji-cuadrada $\left(\chi^{2}\right)$ Mantel-Haenszel, posteriormente se calcularon las razones de momios (RM) e intervalos de confianza (IC) al 95\% (procedimiento FREQ de SAS). Las variables dependientes número de lactaciones, DEL y número de inseminaciones en dependencia de la RP se analizaron con el procedimiento GLM de SAS. La diferencia entre variables independientes (presencia o ausencia de retención placentaria) se evaluó con una prueba de T. Las diferencias se consideraron estadísticamente significativas a un nivel de $p \leq 0.05$.

\section{RESULTADOS Y DISCUSIÓN}

La incidencia total de RP fue de 10.15\% (253 de 2 492). Los factores de riesgo con mayor razón de momios fueron parto gemelar (5.5 veces) y parto prematuro (5.2 veces), seguido de mortinatos (casi cuatro veces) y distocia (casi el doble). La época de parto no estuvo asociada con la presencia de RP. Los factores de riesgo distocia, parto prematuro, parto gemelar y presencia de mortinatos incrementaron la presencia de RP en relación con un parto normal $(p<$ 0.05). Sin embargo, el factor de riesgo época de parto no fue estadísticamente significativo para presencia de RP (Tabla 1).

Como se mencionó, la incidencia total de RP fue del $10.15 \%$, lo que concuerda con el rango reportado por otros autores (Hossein-Zadeh y Ardalan 2011, Hossain et al. 2015, Kumari et al. 2015), que indican que la RP se presenta en un rango de 4 a $18 \%$ de los partos. Esto se puede considerar una incidencia dentro de los límites normales en los establos lecheros, ya que incluso en condiciones normales de gestación y parto, la tasa de RP es de aproximadamente $4.1 \%$ (LeBlanc 2008). De manera similar, un estudio realizado por Melendez et al. (2006) en el estado de Chihuahua, en un contexto climático y genético comparable al de la presente investigación, reportó una incidencia de $7.42 \%$. Por otra parte, Hossain et al. (2015) reportan una ocurrencia del $17.5 \%$, refiriendo que una incidencia mayor puede ser debido a la raza y al pobre manejo en los establos. Con base al análisis de los factores de riesgo evaluados para la presencia de RP, los factores de riesgo con mayor razón de momios se observaron para parto gemelar y parto prematuro. En concordancia, aunque en menor medida, Hossein-Zadeh y Ardalan (2011) reportaron que un parto gemelar incrementa 2.76 veces la probabilidad de que ocurra $\mathrm{RP}$, por lo que concluyen que el parto gemelar es la causa natural más importante para RP en ganado lechero en hatos con buenas prácticas de manejo (Hossein-Zadeh y Ardalan 2011). Al respecto LopezGatius et al. (2005) coinciden con el presente trabajo en el hecho de que la RP se asocia con la presencia de partos gemelares, reportando que $64 \%$ de las vacas con parto gemelar sufrieron RP en contraste con el $15 \%$ de las vacas sin este trastorno.

Los otros factores de riesgo evaluados fueron presencia de mortinato y distocia, que tuvieron razones de momios menores que las anteriores (parto doble y parto prematuro). Estudios realizados en vacas de la raza Holstein (Hossein-Zadeh y Ardalan 2011) y Cebú (Kumari et al. 2015) indican como mayores factores de riesgo al aborto $(\mathrm{RM}=8.46 \mathrm{y}$ 3.9 , respectivamente), mortinatos $(R M=3.18$ y 4.1 , respectivamente) y parto prematuro $(\mathrm{RM}=3.82$ y 4.3 , respectivamente). Contrario a lo esperado y a lo reportado por Hossein-Zadeh y Ardalan (2011, RM = 3.17) y Kumari et al. (2015, $\mathrm{RM}=4.3)$, en el presente estudio la presencia de partos distócicos sólo incrementó al doble la probabilidad de que se presente la RP en relación con un parto normal. Se considera que la distocia es una alteración común en ganado lechero, especialmente en vaquillas primíparas (Yeon-Kyung y III-Hwa 2005). Según los resultados en el presente estudio, la presencia de mortinatos incrementa la incidencia de RP, lo que concuerda con otros autores que han sugerido que la distocia y los mortinatos son causas importantes de RP (Lombard et al. 2007). 
Tabla 1. Factores de riesgo, frecuencias, razón de momios e intervalos de confianza respecto a retención placentaria.

\begin{tabular}{|c|c|c|c|}
\hline Variables & Porcentaje (Frecuencia) & Nivel de significancia & $\mathrm{RM}(\mathrm{IC} 95 \%)^{1}$ \\
\hline Época (partos) & & $p=0.60$ & \\
\hline Otoño & $9.58(70 / 731)$ & & 1 \\
\hline Invierno & $10.76(35 / 325)$ & & $1.14(0.74-1.75)$ \\
\hline Primavera & $11.59(59 / 509)$ & & $1.24(0.86-1.79)$ \\
\hline Verano & $9.60(89 / 927)$ & & $1(0.72-1.39)$ \\
\hline Distocia & & $p<0.0001$ & \\
\hline Ausencia & $8.93(186 / 2,082)$ & & 1 \\
\hline Presencia & $16.38(67 / 410)$ & & $1.99(1.47-2.69)$ \\
\hline Parto prematuro & & $p<0.0001$ & \\
\hline Ausencia & $9.42(228 / 2,420)$ & & 1 \\
\hline Presencia & $34.72(25 / 72)$ & & $5.22(3.15-8.66)$ \\
\hline Parto gemelar & & $p<0.0001$ & \\
\hline Ausencia & $8.90(211 / 2,371)$ & & 1 \\
\hline Presencia & $34.71(42 / 121)$ & & $5.5(3.69-8.23)$ \\
\hline Muerte peri-natal & & $p<0.0001$ & \\
\hline Ausencia & $9.18(217 / 2,363)$ & & 1 \\
\hline Presencia & $27.91(36 / 129)$ & & $3.87(2.57-5.82)$ \\
\hline
\end{tabular}

En cuanto a la presentación de los partos durante las diferentes temporadas del año, en la presente investigación ninguna época se asoció con la presencia de RP, a pesar de la situación climática que enfrentan las vacas en el norte de México, donde los animales están expuestos a una variación de temperatura extrema en diferentes épocas climáticas (calor en verano y frío en invierno). Es necesario considerar que las vacas del establo en el estudio estuvieron alojadas en instalaciones que aminoran las condiciones climáticas extremas, ya que el efecto de los factores de riesgo asociados a RP cambia dependiendo de la variabilidad climática, las prácticas de manejo y las prácticas para el control de la salud del establo (Kumari et al. 2015). Otros autores han reportado mayor incidencia de RP en épocas calurosas (verano), mientras que la menor incidencia se reporta en otoño e invierno (Sheetal et al. 2015, Sharma et al. 2017). Al respecto Labernia et al. (1998) indican que vacas de mayor edad que paren durante temporadas calurosas muestran un riesgo elevado de experimentar RP. Por el contrario, vacas que paren en un ambiente fresco pueden hacer frente de manera más efectiva a los trastornos reproductivos. Esto quizá se deba a que las gestaciones que ocurren en épocas calurosas pueden estar comprometidas por un desarrollo vascular de la placenta anormal y una restricción del crecimiento intrauterino ocasiona- dos por una reducción en la ingesta, provocando un balance energético negativo (Mellado et al. 2016). Sin embargo, otros autores no han encontrado efecto de la época del año en la incidencia de RP, probablemente por efecto de diferencias en la variabilidad climática y las prácticas de manejo en los establos (Han y Kim 2005, Kumari et al. 2015).

En cuanto a distocia, el presente estudio indica una relación importante entre distocia y la presentación de RP derivada de las complicaciones del parto. También se encontró una asociación estrecha entre parto gemelar y RP. La presencia de distocia disminuye el tono muscular uterino y la involución uterina. Además, se presenta un daño al útero por el estrés mecánico resultado de la dificultad al parto, impidiendo el desprendimiento normal de la placenta (Kumari et al. 2015). Asimismo, el aborto y la distocia pueden provocar disturbios en la circulación, afectando la separación de las membranas fetales resultando en RP. De la misma forma, la presencia de mortinatos también predispone a una RP; la aparición de RP más que depender del mismo proceso (mortinato), parece provocar una condición inmunomediada, donde el cuerpo y el sistema inmunológico se ven comprometidos, quedando a expensas de infecciones secundarias, RP, metritis y/o endometritis.

Debido a los hallazgos mencionados en cuanto a la asociación de RP e importantes variables pro- 
Tabla 2. Medias y errores estándar de número de lactaciones, número de inseminaciones a la gestación y días en leche en relación con la presencia de retención placentaria.

\begin{tabular}{lccc}
\hline & \multicolumn{3}{c}{ Variable } \\
\hline & Número de lactaciones & Número de inseminaciones* & Días en leche \\
Retención placentaria & $1.67 \pm 0.10^{a}$ & $3.00 \pm 0.14^{a}$ & $202.25 \pm 7.01^{a}$ \\
Sin retención placentaria & $2.08 \pm 0.03^{b}$ & $3.18 \pm 0.06^{a}$ & $219.03 \pm 2.89^{b}$ \\
\hline$a, b$ Diferentes literales en la misma columna indica diferencias significativas entre presencia o ausencia de \\
retención placentaria $(\mathrm{p}<0.05)$.
\end{tabular}

ductivas y reproductivas, se decidió profundizar en el impacto de la RP sobre el número de lactaciones, número de servicios y días en leche (DEL). La presencia de RP redujo significativamente el número de días en leche y las vacas con más lactancias fueron más propensas a sufrir RP que las vacas con menos lactaciones $(p<0.05)$. Sin embargo, la RP no aumentó el número de inseminaciones necesarias para lograr la gestación (Tabla 2). Otros autores han indicado que, aunque la presencia de RP aumenta el número de servicios por concepción, este incremento no es estadísticamente significativo (Kumari et al. 2015). El nulo impacto de la RP sobre el número de servicios por concepción puede ser debido a que el manejo del establo incluye la aplicación sistémica de antibiótico (penicilina G procaína [200 $000 \mathrm{UI}$ y y dihidroestreptomicina base [164 mg] a una dosis de $20000 \mathrm{UI} \mathrm{kg}{ }^{-1}$ cada $48 \mathrm{~h}$ por tres ocasiones) cuando se diagnostican casos de RP, lo que limita la afectación de RP sobre la fertilidad posterior a la inseminación. Sin embargo, existen reportes que indican que la presencia de RP incrementa el número de servicios por concepción (Melendez et al. 2006, Dawod et al. 2015, Hossain et al. 2015). En este sentido, sería valioso continuar en un futuro la investigación de la posible relación entre el número de inseminaciones y la incidencia de RP. Pese a lo mencionado, la presencia de RP redujo significativamente el número de DEL de las vacas afectadas. Al respecto, Kumari et al. (2015) no encontraron diferencia significativa para DEL entre vacas que presentaron RP y vacas que expulsaron las membranas fetales de manera normal. La reducción en el número de DEL ocasiona una menor cantidad de días en ordeño, situación que puede disminuir la producción láctea, generando afectaciones económicas. Con respecto al número de lactaciones, vacas con mayor número de lactaciones son más propensas a sufrir $\mathrm{RP}$ que vacas con menor número de lactaciones, lo que concuerda con lo reportado en otros estudios, que indican que la incidencia de RP se eleva en vacas de mayor edad, relacionándolo con la disminución del tono de los músculos uterinos conforme la edad avanza (Hossein-Zadeh y Ardalan 2011, Hossain et al. 2015, Kumari et al. 2015, Sharma et al. 2017). Además, las vacas multíparas, al producir más leche en comparación con vacas primíparas, experimentan un balance energético negativo más pronunciado, lo que deprime al sistema inmune, ocasionando que dichos animales sean más susceptibles a desarrollar hipocalcemia, afectando el mecanismo de separación del cotiledón de las carúnculas uterinas (Melendez et al. 2006).

Los factores de riesgo parto gemelar, parto prematuro, mortinatos, distocia y edad de la vaca afectan significativamente la incidencia de retención placentaria, mientras que la época de parto no predispone la presentación de esta alteración. Asimismo, la retención de placenta reduce significativamente los días en leche de la vaca, disminuyendo con esto la producción láctea, pero sin afectar el número de servicios necesarios para lograr la gestación.

\section{LITERATURA CITADA}

Barajas MJL, Hernández CJ, García AA, Martínez BE, Juárez LNO, Bedolla AMA, et al. (2018) Subclinical endometritis and pregnancy rate in dairy cows in Mexico. Revista Mexicana de Ciencias Pecuarias 9: 135- 
146.

Córdova-Izquierdo A, Espinosa CR, Peña BSD, Villa MEA, Huerta CR, Juárez MML, et al. (2017) Effect of placentary retention on open days in cows. Revista Electrónica Veterinaria 18: 1-5.

Dawod A, Mostafa I, El-Baz H, Abdel-Hamid T, Fathala MM (2015) Risks of some postpartum uterine affection on reproduction and milk yield of high yielding dairy cows. Journal of Veterinary Science and Technology 6 : $1-5$.

Grohn Y, Rajala SP (2000) Epidemiology of reproductive performance in dairy cows. Animal Reproduction Science 60: 605-614.

Han YK, Kim IH (2005) Risk factors for retained placenta and the effect of retained placenta on the occurrence of post-partum diseases and subsequent reproductive performance in dairy cows. Journal of Veterinary Science 6: 53-59.

Hossein-Zadeh NG, Ardalan M (2011) Cow-specific risk factors for retained placenta, metritis and clinical mastitis in Holstein cows. Veterinary Research Communication 35: 345-354.

Hossain MK, Billah MN, Aziz SA, Rahman MA, Islam MN, Muslehuddin AHM, et al. (2015) Factors affecting retained fetal membrane and its therapeutic management in dairy cows. International Journal of Natural Sciences 5: 93-97.

Kumari S, Prasad S, Kumaresan A, Manimaran A, Kumar PT, Pathak R, et al. (2015) Risk factors and impact of retained fetal membranes on performance of dairy bovines reared under subtropical conditions. Tropical Animal Health and Production 47: 285-290.

Labernia J, Lopez-Gatius F, Santolaria P, Hanzen C (1998) Influence of calving season on the interactions among reproductive disorders of dairy cows. Animal Science 67: 387-393.

LeBlanc S (2008) Postpartum uterine disease and dairy herd reproductive performance: A review. The Veterinary Journal 176: 102-114.

Lombard J, Garry F, Tomlinson S, Garber L (2007) Impacts of dystocia on health and survival of dairy calves. Dairy Science 90: 1751-1760.

Lopez-Gatius F, García-Ispierto I, Santolaria P, Yaniz J, Nogareda C, Lopez Bejar M (2005) Screening for high fertility in high-producing dairy cows. Theriogenology 65: 1678-1689.

Melendez P, Gonzalez G, Benzaquen M, Risco C, Archbald L (2006) The effect of a monensin controlled-release capsule on the incidence of retained fetal membranes, milk yield and reproductive responses in Holstein cows. Theriogenology 66: 234-241.

Mellado M, López R, de Santiago Á, Veliz FG, Macías-Cruz U, Avendaño-Reyes L, et al. (2016) Climatic conditions, twining and frequency of milking as factors affecting the risk of fetal losses in high-yielding Holstein cows in a hot environment. Tropical Animal Health and Production 48: 1247-1252.

Moretti P, Probo M, Morandi N, Trevisi E, Ferrari A, Minuti A, et al. (2015) Early post-partum hematological changes in Holstein dairy cows with retained placenta. Animal Reproduction Science 152: 17-25.

Sharma M, Bhat Y, Sharma N, Rawat S (2017) Effect of parity of animal and season of year on the rate of retention of placenta in dairy cattle. International Journal of Current Microbiology and Applied Sciences 6: 3103-3108.

Sheetal SK, Choudhary SK, Pandey RP, Sengupta D (2015) Effect of season and parity on incidence of retention of placenta in crossbred cattle. Environment and Ecology 33: 232-234. 
Yeon-Kyung H, III-Hwa K (2005) Risk factors for retained placenta and the effect of retained placenta on the occurrence of postpartum diseases and subsequent reproductive performance in dairy cows. Journal of Veterinary Science 6: 53-59. 
